This item was submitted to Loughborough's Research Repository by the author.

Items in Figshare are protected by copyright, with all rights reserved, unless otherwise indicated.

\title{
Foundations of atmospheric pressure non-equilibrium plasmas
}

PLEASE CITE THE PUBLISHED VERSION

https://doi.org/10.1088/1361-6595/aa97af

PUBLISHER

(c) IOP Publishing

VERSION

AM (Accepted Manuscript)

LICENCE

CC BY-NC-ND 4.0

REPOSITORY RECORD

Bruggeman, Peter, Felipe Iza, and Ronny Brandenburg. 2019. "Foundations of Atmospheric Pressure Nonequilibrium Plasmas". figshare. https://hdl.handle.net/2134/27605. 


\title{
Foundations of high pressure non-equilibrium plasmas
}

\author{
Peter J. Bruggeman ${ }^{1}$, Felipe Iza $^{2}$, Ronny Brandenburg ${ }^{3}$ \\ ${ }^{1}$ University of Minnesota, Department of Mechanical Engineering, 111 Church Street \\ SE, Minneapolis, MN 55455, USA \\ ${ }^{2}$ Wolfson School of Mechanical, Electrical and Manufacturing Engineering, \\ Loughborough University, Loughborough LE11 3TU, United Kingdom \\ ${ }^{3}$ Leibniz Institute for Plasma Science and Technology (INP Greifswald), \\ Felix-Hausdorff-Strasse 2, D-17489 Greifswald, Germany \\ E-mail: pbruggem@umn.edu
}

\begin{abstract}
Non-equilibrium plasmas have been intensively studied over the past century in the context of material processing, environmental remediation, ozone generation, excimer lamps and plasma display panels. Research on high-pressure nonequilibrium plasmas intensified over the last two decades leading to a large variety of atmospheric-pressure plasma sources that have been developed for an extended application range including chemical conversion, medicine, chemical analysis and disinfection. The fundamental understanding of these discharges is emerging but there remain a lot of unexplained phenomena in these intrinsically complex plasmas. The properties of non-equilibrium plasmas at atmospheric pressure span over a huge range of electron densities as well as heavy particle and electron temperatures. This paper provides an overview of the key underlying processes that are important for the generation and stabilization of high-pressure non-equilibrium plasmas. The unique physical and chemical properties of theses discharges are also summarized.
\end{abstract}




\section{Introduction}

High-pressure non-equilibrium (or non-thermal) plasmas have a long history dating back to the XIX century. Dielectric barrier discharges and corona discharges, for example, have been used in applications such as ozone generation, gas purification, electrostatic precipitation, combustion and surface functionalization of materials for decades $[1,2]$.

As they do not require expensive vacuum systems, high-pressure and more precisely atmospheric-pressure plasmas are often proposed as a cost-effective alternative to existing low-pressure plasma processes. While this is certainly valid for some applications, it is an oversimplistic and incomplete view of high-pressure plasmas that certainly does not apply to all plasma processes. Atmospheric-pressure plasmas present unique opportunities but also face important challenges when compared with their lowpressure counterparts.

In recent years, high-pressure non-equilibrium plasmas have received renewed attention in large part motivated by their ability to treat liquids and heat sensitive materials, such as biological targets, that cannot withstand low pressure or vacuum environments. These new treatments have given raise to exciting applications of nonequilibrium plasmas in medicine, disinfection, water treatment, nanoparticle synthesis, food processing and agriculture [3]. Many different plasma generation methods have been devised to address the different requirements of these applications. The properties of the resulting high-pressure plasmas spread across order of magnitudes in terms of size, time scales, temperatures as well as charged and reactive species densities. Figure 1 provides an overview of the range of electron densities and gas temperatures that are encountered in typical high-pressure non-equilibrium plasmas.

As it is often the case in the literature, in this paper the words plasma and gas discharge are used interchangeably. However, given the exceptionally large range of operational parameters encountered in high-pressure discharges, it is not surprising that some of these discharges fail to develop a quasi-neutral bulk and therefore are not strictly speaking plasmas. This is definitely the case for discharges where the characteristic size of the discharge is comparable to the plasma sheath width $(10-100 \mathrm{~s} \mu \mathrm{m})$ or when the discharge is highly transient. For example, ions, due to their large inertia, remain practically static in nanosecond pulsed discharges while electrons drift causing significant charge separation. The modelling of some of these discharges using conventional fluid descriptions is problematic as some of the intrinsic fluid assumptions break down.

In non-equilibrium plasmas the mean kinetic energy of electrons is larger than the gas temperature $\left(T_{e}>>T_{g}\right)$. Maintaining this non-equilibrium character is possible because energy transfer from the applied electric field to the electrons is generally much faster and efficient than the subsequent collisional energy transfer between electrons and heavy particles [4]. This latter process is slow due to the large mass difference between electrons and heavy particles. For a steady-state plasma sustained in an atomic gas, the gas temperature $T_{g}$ can be estimated by balancing the energy electrons gain in an electric field $E$ with the energy they lose through elastic collisions with background 


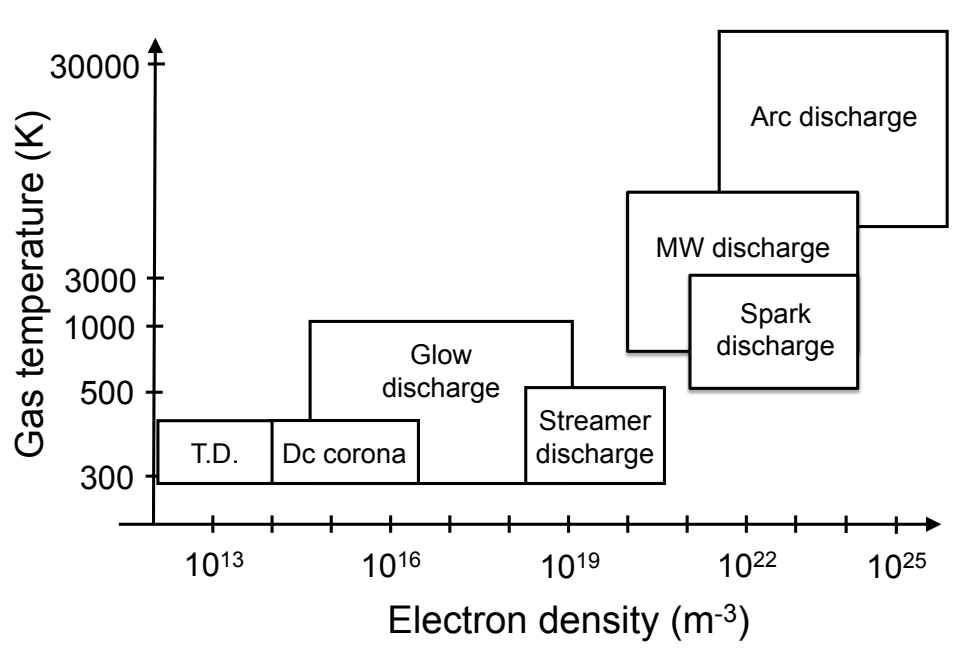

Figure 1. Overview of different high-pressure gas discharges spanning a range of more than 10 orders of magnitude in electron density. T.D. stands for Townsend discharge.

neutrals $[5,6]$ :

$$
T_{g}=T_{e}\left(1-\frac{m_{g}}{4 m_{e}} \frac{\left(\lambda_{e} e E\right)^{2}}{\mathcal{E}_{e}^{2}}\right)
$$

where $m_{g}$ is the heavy neutral particle mass, $m_{e}$ the electron mass, $\mathcal{E}_{e}$ the electron energy, $e$ the elementary charge and $\lambda_{e}$ the electron mean free path. Equation 1 shows that maintaining moderate gas temperatures becomes increasingly challenging at high pressure due to the square dependence on the electron mean free path. The high collisionality encountered in high-pressure plasmas not only leads to increased gas heating but it also enhances the tendency to develop spatio-temporal instabilities. Without the use of special plasma excitation methods and/or special plasma electrode geometries, high-temperature (thermal) discharges, such as arcs, will form at atmospheric pressure.

For atmospheric-pressure plasma applications where a gas temperature increase is not acceptable, a number of strategies have been developed to minimize gas heating. These include the use of noble gases (higher thermal conductivity and lower inception voltage than most molecular gases), gas flows, reduced plasma size (increased surface to volume ratio), and transient operation of the discharge. An overview of these different approaches and their rationale is provided in subsequent sections. Although non-equilibrium plasmas in which the gas remains close to room temperature have been reported, developing stable non-equilibrium homogeneous high-pressure plasmas remains the focus of active research.

Most applications of high-pressure non-equilibrium discharges rely on their unique non-equilibrium plasma chemistry. This chemistry is driven by energetic electrons 
(rather than thermal energy) and can be used to initiate and catalyze chemical processes at moderate gas temperatures. This means that with controlling the electron energy distribution function (EEDF) in the plasma one can tune the plasma reactivity. However, the EEDF depends not only on the applied electric field but also on the space charge and the evolving plasma chemistry, and as a result controlling the chemistry in these plasmas is challenging and remains an area of active research.

The rest of the paper is structured as follows. Section 2 describes scaling laws and time scales of important plasma processes that are relevant to discharge generation and instability development, which are ubiquitous in high-pressure discharges. Section 3 highlights unique properties of high-pressure plasmas and ionization processes leading to breakdown. Common discharge instabilities are discussed in sections 4 and 5 . Section 6 discusses different approaches typically employed to stabilize and maintain atmopspheric-pressure plasmas and conclusions and an outlook are presented in section 7.

The paper is an introductory overview but not exhaustive. For more in depth information it refers to comprehensive review articles and textbooks. In particular, a detailed description of many aspects of the physics and chemistry of high-pressure non-equilibrium plasmas can be found in $[7,8,9,10,11,12,13,14]$.

\section{Scaling laws and time scales}

Scaling laws are useful proportionality relations that can be used to generalize results obtained at various scales and to provide insights into the origin of fundamental differences between plasma sources. Here the physical meaning of a few scaling parameters relevant to non-equilibrium plasmas are discussed and used to highlight the unique nature of high-pressure discharges. Time scales of fundamental processes that are crucial for the understanding of discharge inception, breakdown, plasma kinetics, plasma stability and controllability of plasmas are also discussed.

Arguably the most famous scaling law in plasma physics is Paschen's law. It states that the voltage necessary to ignite a plasma between two electrodes for a given background gas depends on the product of pressure $(p)$ times electrode gap distance (d) [16]. The reason behind this experimental observation is that the inception voltage is underpinned by a balance between the generation of electrons in volumetric electron avalanches and secondary electron emission processes and the loss of electrons on the surfaces, which for a DC breakdown ultimately depends on the number of collisions electrons undergo as they transit between the electrodes. This number is proportional to the product of the gas density $(n)$ and the electrode gap distance. Since at inception the gas is nearly at room temperature, $n$ is proportional to $p$ and hence the inception voltage is typically reported as a function of $p d$. Paschen curves, i.e. plots of the breakdown voltage versus $p d$ values, have a similar shape for different gases with a minimum at $p d$ values in the range of 1 to 10 Torr-cm $[4,7,16]$. The increase in breakdown voltage at lower $p d$ values is due to the reduced ionization and increased loss 
of electrons that occurs as a result of the electron mean free path becoming comparable to the gap between the electrodes. At large $p d$ values, the breakdown voltage increases as the energy electrons gain in between collisions for a given applied voltage decreases due to the increasing number of collisions. Most atmospheric-pressure plasmas operate in this latter regime $(p d>10$ Torr-cm) although atmospheric-pressure microplasmas (see further) take advantage of the scaling and are operated closer to the Paschen minimum.

Despite its broad applicability, deviations from the Paschen law have been reported and reflect changes in the underpinning mechanisms governing the breakdown. The onset of field emission in small gaps and the trapping of electrons in an oscillatory motion between the electrodes in radio-frequency $(\mathrm{RF})$ discharges are examples of known mechanisms that can cause such deviations [14, 17, 18].

Besides the $p d$ product, other important scaling parameters often used in the study of non-equilibrium plasmas include $E / n$ and $\omega_{r f} / \nu$, where $E$ is the applied electric field, $n$ the gas density, $\omega_{r f}$ the (angular) frequency of the applied voltage and $\nu$ the electron-neutral collision frequency. The reduced electric field strength $E / n$ is a measure of the energy that electrons gain from the electric field in between collisions. The first Townsend ionization coefficient $\alpha$ and other parameters depending on the mean electron energy scale with $E / n$, which is typically given in the unit Townsend $\left(\mathrm{Td}=10^{-17}\right.$ $\mathrm{Vcm}^{-2}$ ). The scaling parameter $\omega_{r f} / \nu$ is of interest when comparing discharges that operate at different frequencies and/or pressures as it relates to the number of collisions electrons undergo in one RF cycle. For electrons to gain energy from an alternating field, collisions are needed and for a given field amplitude, it can be shown that maximum power transfer from the electric field to the electrons occurs when $\omega_{r f} / \nu=1[4]$. In atmospheric-pressure discharges the electron-neutral collision frequency is typically in the order of $\mathrm{THz}$ and as a result these discharges operate in a regime that is far from this optimum even when sustained at microwave frequencies.

Table 1 compares a low-pressure and an atmospheric-pressure RF plasma. It is important to note that not only some plasma parameters are different but actually that the scaling parameters differ by orders of magnitude. This indicates that atmosphericpressure non-equilibrium plasmas represent a different paradigm than their low-pressure counterparts. Some of the unique aspects of high-pressure plasmas are further discussed in section 3 .

Time scales for collisional processes and transport in non-equilibrium atmosphericpressure plasmas span over 12 orders of magnitude. An overview of the most important plasma processes with their typical time scales is shown in Figure 2. Electron collisional processes typically take place in picosecond time scales with discharge inception, excitation, dissociation reactions and ionization processes being observed at nanosecond time scales. The large collisionality of atmospheric-pressure discharges also leads to short energy relaxation times, which tend to drive electron kinetics in equilibrium with the instantaneous electric field. For example, in helium atmospheric-pressure plasmas, the energy relaxation time for low-energy and high-energy electrons with energies below and above the helium excitation threshold is on the order of nanoseconds and picoseconds, 


\begin{tabular}{|c|c|c|}
\hline Parameter & He low pressure plasma & He atmospheric-pressure plasma \\
\hline Pressure $(p)$ & $100 \mathrm{mTorr}$ & 760 Torr \\
\hline Gas density $(n)$ & $3.2 \cdot 10^{15} \mathrm{~cm}^{-3}$ & $2.5 \cdot 10^{19} \mathrm{~cm}^{-3}$ \\
\hline Electrode gap distance $(d)$ & $10 \mathrm{~cm}$ & $1 \mathrm{~cm}$ \\
\hline Angular excitation frequency $(\omega)$ & $13.56 \mathrm{MHz}$ & $13.56 \mathrm{MHz}$ \\
\hline Electron temperature $\left(T_{e}\right)$ & $3 \mathrm{eV}$ & $1 \mathrm{THz}$ \\
\hline Electron-neutral collision frequency $(\nu)$ & $150 \mathrm{MHz}$ & $\sim 760$ Torr $\mathrm{cm}\left(>>p d_{\text {min }}\right)$ \\
\hline$p d$ & $\sim 1$ Torr $\mathrm{cm}\left(\leq p d_{\min }\right)$ & $10-100 \mathrm{Td}$ \\
\hline$E / n$ & $10-100 \mathrm{Td}$ & $10^{-5}(<<1)$ \\
\hline$\omega_{r f} / \nu$ & $\sim 0.1$ & \\
\hline
\end{tabular}

Table 1. Comparison of a low pressure and an atmospheric pressure RF plasma in helium. Differences in the scaling parameters highlight the different paradigms of the two non-equilibrium plasma discharges [19].

respectively; time scales shorter than the typical period of the applied voltage waveform. As a result, plasma properties such as electron temperature and ionization rates display strong periodic oscillations even in high-frequency atmospheric RF discharges.

Although individual electron-neutral elastic collisions result in little energy transfer due to the disparity in the masses of electrons and heavy neutrals, the large collisionality encountered in atmospheric-pressure plasmas $(\nu \sim \mathrm{THz})$ can result in significant power transfer by elastic collisions to the background gas. Gas heating through elastic collisions (and vibrational excitation in the case of molecular gases) typically takes between $100 \mathrm{~ns}$ to $1 \mu \mathrm{s}$. Depending on the plasma conditions, faster gas heating can also take place via the recombination of highly excited atoms and molecules, as observed for example in nanosecond pulsed discharges in air [20]. Gas heating does not only represent an undesired energy loss for many applications but it can also drive thermal instabilities. In addition, fast gas heating on nanosecond time scales can also lead to sudden increases in pressure and the creation of shock waves [21].

Ionic recombination reactions and metastable induced dissociation reactions, although highly dependent on the electron density of the plasma, typically occur on an intermediate timescale from 10 s of nanoseconds to microseconds. Neutral (radical) induced reactions are typically slower and proceed on a microsecond to millisecond time scale. The coupling of transport through diffusion and convection takes typically between 10s of microseconds up to several seconds and couples mainly with neutral chemistry and can influence pre-ionization in modulated and repetitively pulsed discharges (see further).

\section{Unique aspects of high-pressure plasmas}

As shown in Table 1, non-equilibrium atmospheric-pressure plasmas operate in a very different parameter space than their low-pressure counterparts and as a result, they 
Figure 2. Timescales of relevant collisional and transport processes in high-pressure plasmas.

offer new possibilities and present their own limitations. Arguably, the most important characteristic of low-pressure non-equilibrium plasmas is their ability to drive ions anisotropically against a target substrate. This anisotropic bombardment is possible because at low-pressure the ion mean free path is larger than the plasma sheath width [4]. In the past decades, significant efforts have been made to fine tune and control the ion energy distribution function (IEDF) of ions impinging onto the substrates in order to improve the resolution and repeatability of different low-pressure plasma treatments. In general, the IEDF depends on the sheath potential, the sheath width, the ion mean free path, the ion mass and its transit time as compared to the applied RF period. Experimentally these parameters can be influenced by careful selection of the background gas pressure (ion mean free path), gas composition (ion mass) and applied voltage (sheath potential and sheath width) [22].

As shown in Figure 3, the energy of the ions hitting a substrate decreases rapidly with increasing pressure. For the particular case depicted in figure 3, the maximum ion energy decreases from $\sim 1000 \mathrm{eV}$ at $30 \mathrm{mTorr}$ (4 Pa), to a few hundred $\mathrm{eV}$ at 75 mTorr $(10 \mathrm{~Pa})$ and to a few tens of eV at $750 \mathrm{mTorr}(100 \mathrm{~Pa})$. Ion mean free paths at atmospheric pressure are on the order of $\sim 1 \mu \mathrm{m}$ and therefore hundreds of times smaller than typically sheath widths. As a result, although physical bombardment is critical to the success of many low-pressure plasma processes, the high collisionality encountered at atmospheric pressure prevents the collisionless transition of ions across the sheath and as inferred from the trend shown in figure 3, ions reaching the target do so with very little energy [19]. In streamer-based discharges high-energy ions can reach the electrode when streamers reach the electrode, but their fluxes are typically small. Therefore, one can say that low-pressure plasma processes that rely on anisotropic ion bombardment 


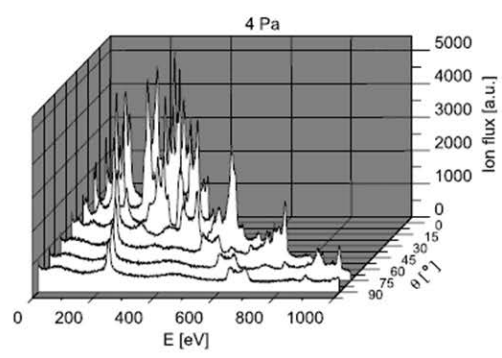

(a)

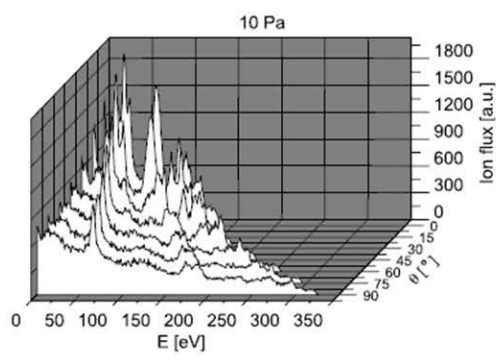

(b)

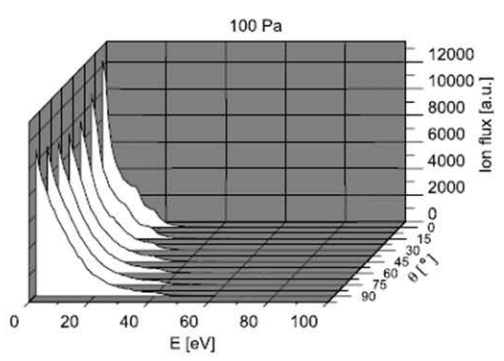

(c)

Figure 3. Ion energy distribution functions at the ground electrode of a dual frequency capacitively coupled RF plasma with an electrode gap of $2 \mathrm{~cm}$ operated at three pressures: (a) 30, (b) 75 and (c) $750 \mathrm{mTorr}$. The angle $\theta$ is the phase difference between the two harmonics used to create an electrical asymmetry effect (13.56 and 27.12 MHz). Reprinted from [23], with permission of IOP Publishing.

are not easily transferable to a similar atmospheric-pressure process.

Although magnetic confinement has been exploited in low-pressure plasmas to improve plasma efficiency, the numerous collisions that electrons undergo in a cyclotron period disrupt any effective confinement at atmospheric pressure. Additionally, collisionless electron heating by oscillating sheaths can also be safely neglected in atmospheric-pressure discharges as even for small microdischarges the product of the collision frequency times the gap distance is much larger than the electron thermal velocity $\nu d>>v_{t h}[4]$. The lack of stochastic heating directly impacts on the electron energy distribution function and bi-Maxwellian distributions often encountered in lowpressure RF plasmas are not found in their atmospheric-pressure counterparts.

Nonetheless the increased collisionality in atmospheric-pressure plasmas brings about many interesting features. For example, collisions between ions and neutral background species result in effective energy and momentum transfer, a feature that has been exploited in plasma actuators to modify the laminar to turbulent transition inside boundary layers and to prevent or to induce flow separation [24]. In addition, this interaction often referred to as electrohydrodynamic (EHD) effect can be exploited to create synthetic jets that are perpendicular to a discharge surface, thereby enhancing the transport of reactive species from a surface discharge to the substrate being treated [25]. Figure 4a shows the formation of one such a synthetic jet by an annular dielectric barrier discharge [26]. The interplay between plasma and gas dynamics is in general quite complex and in the same way that plasma can affect flow dynamics, flow conditions also influence the plasma kinetics and formation [27]. Figure $4 \mathrm{~b}$ shows 4 jets operating in parallel in which the presence of plasma triggers a flow instability that enhances the entrainment of the surrounding air and the jets repel each other to a different degree depending on the neutral gas flow rate. As shown in Figure 4c, in pulsed discharges rapid pressure variations and shockwaves can occur on submicrosecond timescales, which further complicates the interplay between plasmas and flows [29, 21].

Since, as discussed above, physical ion bombardment is not very energetic 


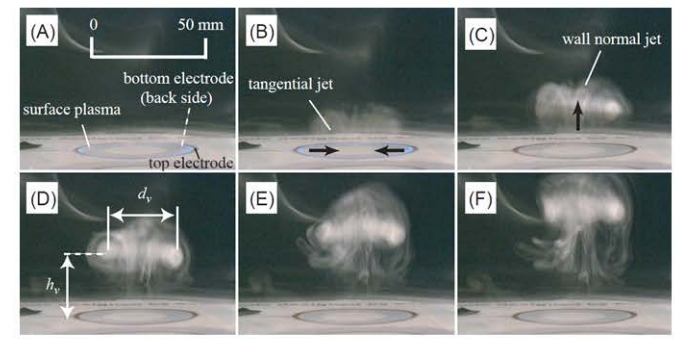

(a)

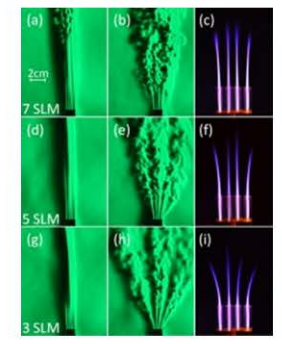

(b)

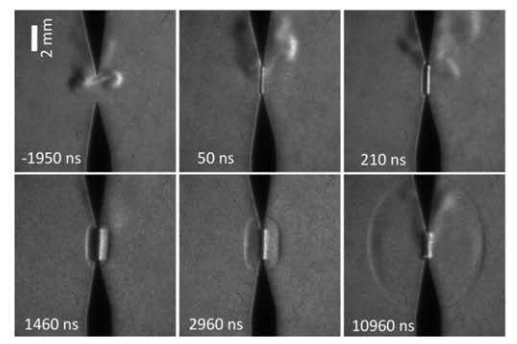

(c)

Figure 4. Examples of the interaction between plasmas and gas flows. (a) Flow visualization using smoke of a synthetic jet perpendicular to a surface induced by an annular dielectric barrier discharge. Reprinted from [26], with permission of ASME. (b) Schlieren images of 4 plasma jets interacting at different gas flow rates. Reprinted from [28], with permission of IOP Publishing. (c) Schlieren images (20ns exposure time) of the time evolution of a pin-to-pin pulsed plasma in air showing the launch of a shockwave. Reprinted from [29], with permission of AIP Publishing.

in atmospheric-pressure plasmas due to ion-neutral collisions in the sheath, most atmospheric-pressure treatments rely on plasma chemistry. Here again, the increased background density brings about differences with respect to the processes observed in low-pressure plasmas and in particular, 3-body collisions become important. In atmospheric-pressure plasmas the collision rates of 3-body processes are four to six orders of magnitude larger than in conventional low-pressure plasmas. As a result, atmospheric-pressure discharges of atomic noble gases present significant concentrations of dimer ions and molecules including excimers which lead to the generation of VUV radiation $[2,81]$. The 3 -body collisions also lead to the formation of chemical species such as $\mathrm{O}_{3}$ and $\mathrm{H}_{2} \mathrm{O}_{2}$, which at low pressure can only be produced through surface reactions. In additon, in the presence of water molecules, positive and negative ions in atmospheric-pressure plasmas become hydrated, with larger water clusters at higher water content. This hydration can significantly impact the ion mobility and affect the overall plasma dynamics [30].

Although plasma diagnostics fall beyond the scope of this paper, high collisionality causes additional difficulties in the implementation and/or interpretation of conventional plasma diagnostics. For example, the use of a mass spectrometer requires multi-stage differential pumping, optical (laser) diagnostics and stoichiometric methods need to account for often complex collisional transfer processes, and no universally theory exists for the interpretation of Langmuir probe measurements at atmospheric pressure $[31,32,33,34]$.

\section{Discharge inception and breakdown mechanisms}

The inception of non-equilibrium plasmas and gas discharges is based on the development of electron avalanches. The very first free electrons, which are starting the discharge inception, are provided by cosmic rays, radioactivity or leftover charges 
from previous discharge activity. The main ionization mechanism in most gases is direct electron impact ionization by free electrons accelerated in the applied electric field $E$. In particular, at low pressure the positive ions created in the ionization process drift to the cathode and lead to secondary emission of electrons. This subsequent delivery of free electrons seeds new avalanches and this is the main aspect of the Townsend-breakdown mechanism that leads to the generation of a self-sustained gas discharge. The process can be thought of as successive avalanches crossing the discharge gap without the build up of space charge. The voltage at which a self-sustained discharge is obtained is described by the Paschen law and it is a function of the scaling parameter $p d$ as discussed in section 2 .

At higher pressure and thus higher gas density $n$, the number of ionizing collision per unit volume increases and the breakdown mechanism changes significantly. In particular, when electron avalanches create a space charge that locally enhances the applied electric field, secondary electron avalanches are triggered in the gas phase $[7,10,15]$. These are mostly initiated by photo-ionization or (in the case of sufficient electric field strength in electronegative gases) by electron detachment from negative ions previously formed by electron attachment on electronegative molecular species. The ionized region and its perturbation of the electric field grows rapidly and finally a distinct plasma channel, the so-called streamer, is formed. In air and other molecular gases at atmospheric pressure streamer formation occurs on a time scale of nanoseconds. Figure 5a depicts schematically the formation of a positive or cathode-directed streamer. Negative or anode directed streamers also exist and are very similar to positive streamers although the secondary avalanches start at the streamer head and grow towards the anode.

While the Townsend-criterion provides the inception voltage for the Townsend mechanism, the Meek criterion (sometimes also referred too as the Raether criterion) describes the conditions for streamer initiation. The streamer formation conditions relate to the charge number density in the primary avalanche required for significant space charge field perturbation, namely $\exp \left[\alpha_{\text {eff }} \cdot d\right]=10^{8}$, where $\alpha_{\text {eff }}$ is the effective first Townsend ionization coefficient. In air, this criterion is typically fulfilled at $p d$ values above 1000 Torr-cm.

Streamers can be considered as the initial stage of the electric breakdown of any non-ionized medium (gases, liquids and solids). They can precede sparks, create the path for leaders in lightnings and are responsible for the filamentary discharge structure in many non-equilibrium plasmas at atmospheric pressure. Although cathode as well as anode directed streamers have been observed, cathode directed (or positive) streamers are more prominent and anode directed (negative) streamers are only obtained in the case of high over-voltages and sufficiently large discharge gaps. Both types of streamers can exhibit branching and thus, rather complex spatial structures [35].

The interior of a streamer consists of a conducting, roughly quasi-neutral plasma while a thin and curved space charge layer at its tip is responsible for the screening of the inner ionized area and the strong field enhancement at the streamer head. Electrons in the field enhanced zone around the streamer head can be highly energetic and due 


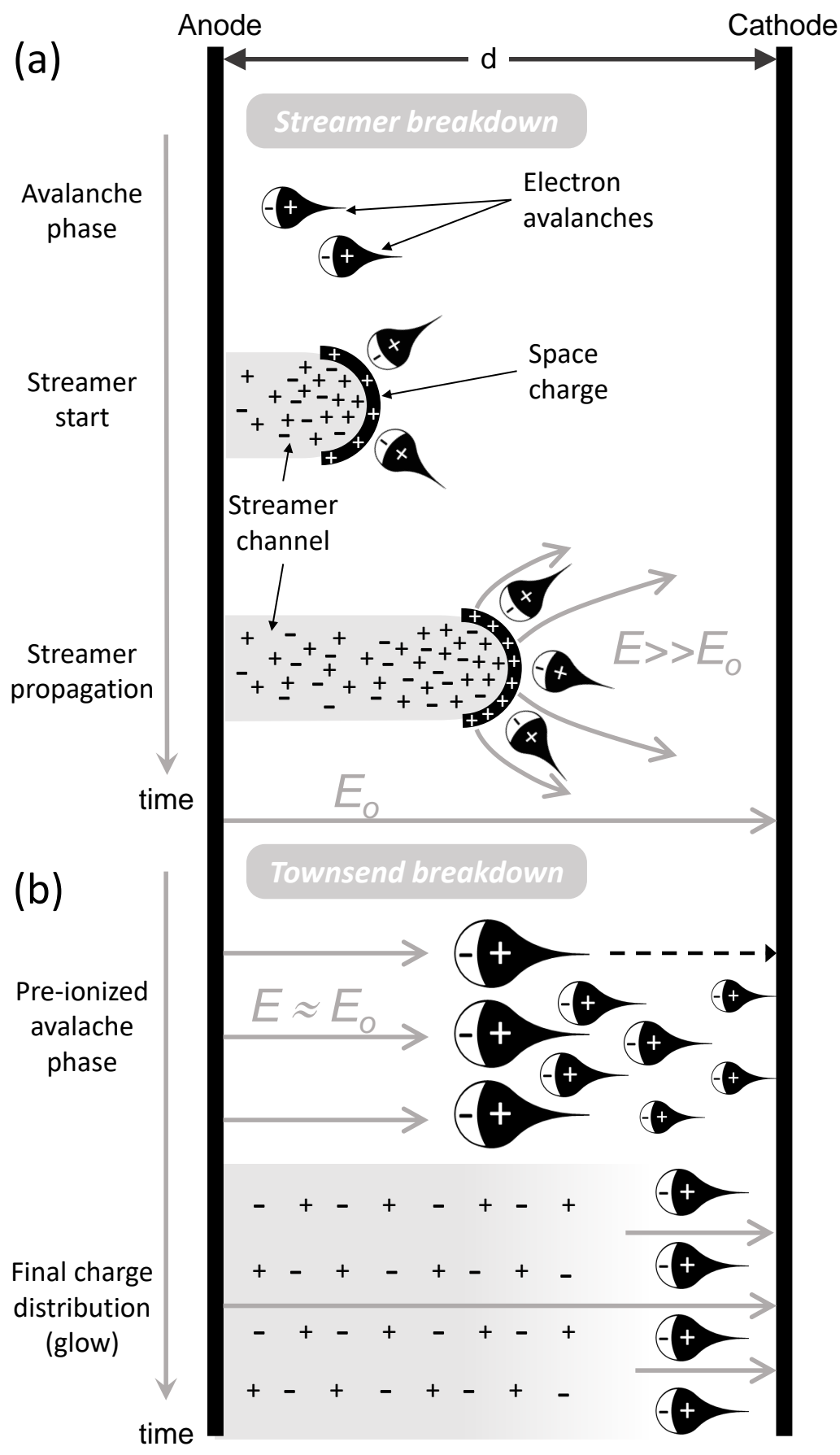

Figure 5. Development of a positive streamer due to electron avalanches and space charge formation (a) and Townsend-breakdown in case of sufficient pre-ionization (b).

to the field enhancement, streamers can propagate into areas where the background electric field is below the ionization threshold of the background gas.

The tendency to form streamers is related to the dependence of the effective reduced Townsend ionization coefficient on the reduced electric field and in particular $\partial\left(\alpha_{e f f} / n\right) / \partial(E / n)$, as this determines the increase of the ionization frequency with 
changes in local electric field strength. Helium or neon are characterized by a relatively high ionization coefficient at a relative low electric field strength and a small gradient $\partial\left(\alpha_{e f f} / n\right) / \partial(E / n)$ compared to argon and most molecular gases or gas mixtures. Hence in gases such as helium or neon, the rapid formation of field gradients is suppressed and it is easier to obtain a diffuse breakdown [36].

The streamer development as described above requires the development of a strong local space-charge gradient that can lead to sufficient field enhancement as to cause the formation of the streamer. This condition is readily achieveable when the discharge starts with a single or few electrons leading to well separated avalanches that do not greatly influence each other. However, when the background pre-ionization density is larger, avalanches influence each other and overlap. The overlap of these avalanches smoothes out local gradients in the space-charge field and leads to a more homogeneous charge density. Therefore, a sufficiently high level of pre-ionization is a means to inhibit streamer formation. Figure 5b schematically depicts the Townsend breakdown in the presence of pre-ionization.

A typical background ionization density in air due to the presence of low levels of radioactive materials and cosmic radiation is of the order of $10^{3}-10^{4} \mathrm{~cm}^{-3}$ [37]. The pre-ionization density required for suppression of the Meek condition and therefore the formation of a diffusive breakdown can be estimated by imposing that the distance between avalanches is comparable to the typical radius of the avalanche at streamer formation, which is of the order of $100 \mu \mathrm{m}$ in air [38]. Estimating the distance between avalanches by the pre-ionization density $\left(\propto n_{0}^{-1 / 3}\right)$, pre-ionization densities in excess of $10^{6} \mathrm{~cm}^{-3}$ are typically required for diffuse breakdown at atmospheric pressure. This preionization can be achieved by an electron beam or electrons remaining in the discharge gap from a (potentially non-uniform) discharge used to pre-ionize the discharge gap (see also section 6$)$.

As mentioned earlier, the breakdown at atmospheric pressure proceeds on a timescale of typically several tens of nanoseconds. Changing the applied voltage on the same timescale as the discharge generation by short (usually sub-microsecond) high voltage pulses having a rise time of $100 \mathrm{~V} / \mathrm{ns}$ or higher and combined with a high overvoltage (i.e. applied voltage amplitude exceeding the Paschen-breakdown voltage significantly) allows one to strongly impact the discharge formation.

The creation of non-equilibrium plasmas in even denser media including liquids, particularly in conductive liquids, requires the application of short high voltage pulses. While it is possible to ignite discharges in liquids with continuous or sinusoidal voltages with moderate frequency, such plasma formation is always coinciding with a phase change or formation of gas/vapor bubbles [39, 40]. It is however much easier to break down liquids with short high-voltage pulses when the pulse duration is shorter than the dielectric relaxation time of the liquid. In this case the liquid behaves as a dielectric rather than a conductive medium. Many studies have investigated breakdown processes in liquids including liquid $\mathrm{Ar}, \mathrm{N}_{2}$, hydrocarbons and water and the reduced Townsend ionization coefficient $\alpha / n$ does typically not scale as in the equivalent gas 
phase [41]. While not all details are currently understood, the higher density can lead to stepwise ionization processes and two-body collision approximations used in the gas phase breakdown theory are no longer valid in the liquid phase.

\section{Discharge transitions and instabilities}

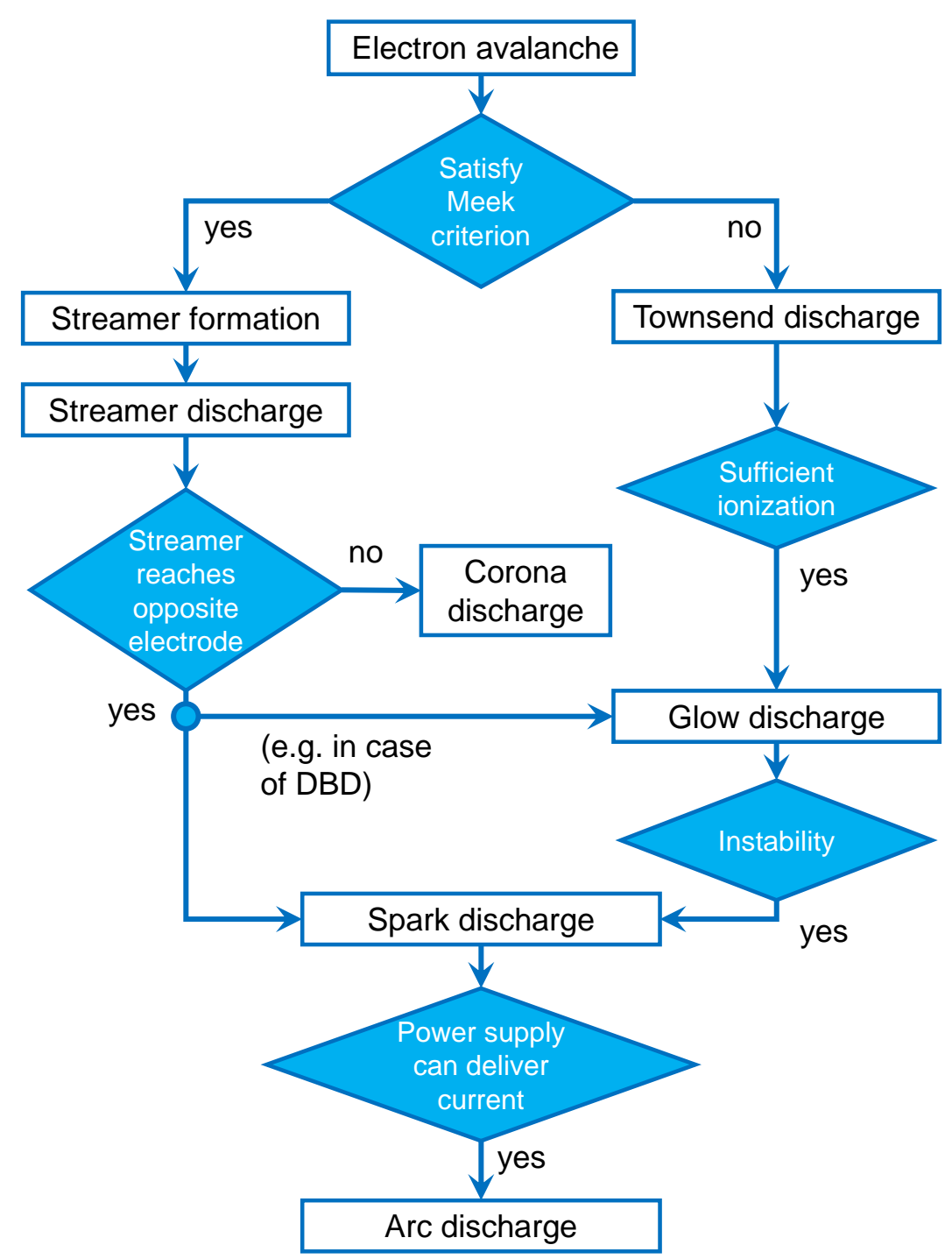

Figure 6. Overview of the different transitions that occur in high-pressure plasma discharges.

In the previous section we have discussed the transition from an avalanche to a streamer as a result of the build-up of space charge in the discharge volume. Other transitions exist and these are often a result of a plasma instability. An overview of typical transitions in high-pressure plasma discharges is shown in Figure 6. Diffuse discharges are obtained when the ignition conditions do not satisfy the Meek criterion. At atmospheric-pressure, however, these discharges are prone to instabilities that can 
trigger the transition from a glow to a spark discharge. Instabilities can be driven by several mechanisms but they always relate to an unbalance between the ionization and the recombination rate of electrons, leading to excessive ionization [7]. This can be mathematically expressed as:

$$
\frac{d n_{e}}{d t}=k_{i o n}\left(T_{e}\right) n_{e} n+\ldots-\frac{D_{a}}{\Lambda^{2}} n_{e}-k_{d r} n_{e}^{2}-\ldots>>0
$$

where $k_{i o n}$ is the electron impact ionization rate coefficient, $D_{a}$ the ambipolar diffusion coefficient, $\Lambda$ the diffusion length and $k_{d r}$ the electron-ion dissociative recombination rate. Many other reactions including attachment, detachment, Penning ionization processes and 3-body electron recombination processes could also play a role hence the ellipsis in equation 2. Equation 2 shows that in most cases an increase in the electron density does not lead on itself to runaway behavior as dissociative electronion recombination, the dominant electron recombination process in many high-pressure plasmas, scales quadratically with $n_{e}$ while the ionization scales only linearly with $n_{e}$. However, a sudden change in the electron temperature can trigger an instability due to the exponential dependence of the ionization rate coefficient on the electron temperature. This non-linear dependence of the electron production rate on a changing plasma parameter is necessary to obtain an instability. In most cases, fluctuations in the gas temperature and electron temperature lead to an unbalance in the electron production and recombination, causing the onset of the instability. The ionization rate in equation 2 scales with the neutral gas density $n$ and therefore, the development of an instability is much faster in high-pressure plasmas than in their low-pressure counterparts. An example of the development of an instability in a pulsed atmosphericpressure air glow discharge is shown in Figure 7.

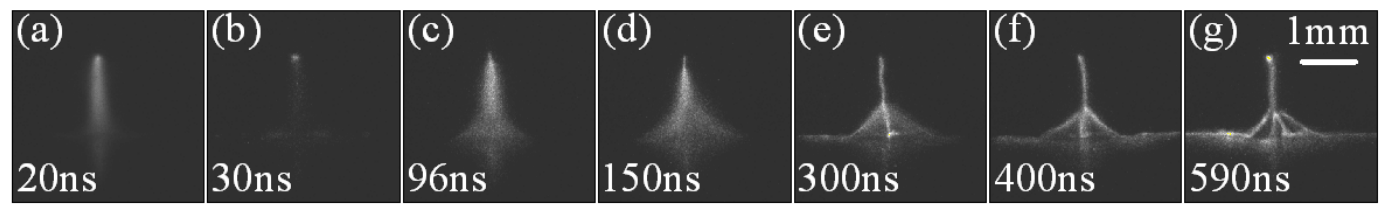

Figure 7. Example of the development of an instability in a nanosecond pulsed glow discharge in air between a metal pin electrode and a liquid surface with liquid electrode. Reprinted from [42] with permission of IOP Publishing.

The most common instability is the so-called thermal instability. This instability is triggered by small fluctuations in the electron density that lead to the following chain of events. An increase in electron density leads to increased Joule heating and thereby a localized increase in the gas temperature and decrease in gas background density. Assuming that the electric field in the positive column of a glow discharge remains constant, $E / n$ will then increase, causing an increase in $T_{e}$. The increase in $T_{e}$ leads to an increased ionization frequency $\left(\nu_{e}\right)$, which further increases $n_{e}$, leading to positive feedback and unstable behavior. The cycle leading to the instability can be summarized 
as follows:

$$
\delta n_{e} \uparrow \rightarrow \delta T_{g} \uparrow \rightarrow \delta\left(\frac{E}{n}\right) \uparrow \rightarrow \delta T_{e} \uparrow \rightarrow \delta \nu_{e} \uparrow \rightarrow \delta n_{e} \uparrow
$$

Runaway behavior of the ionization rate can also occur due to stepwise ionization. The ionization rate coefficient of metastable species, particularly in noble gases are orders of magnitude larger than direct ionization of the ground state species. Hence, when the density of metastable species reach a critical value, the direct ionization is taken over by stepwise ionization. This can lead to a sudden increase of the ionization rate. Instabilities often occur when the dominant ion is a dimer such as in a highpressure $\mathrm{Ar}$ discharge. In the case of $\mathrm{Ar}$, the electron-ion recombination of $\mathrm{Ar}_{2}^{+}$leads to the production of an Ar metastable atom which is readily re-ionized to $\mathrm{Ar}^{+}$. As the formation of $\mathrm{Ar}_{2}^{+}$from $\mathrm{Ar}$ is very fast at atmospheric pressure, intrinsically a very fast recycling between the ion and the metastable occurs which is prone to trigger instabilities. Further considerations about the relation between these reactions and the contraction of a discharge channel in an atmospheric-pressure microwave discharge can be found in [43].

Another cause of instability can be encountered when the dominant electron loss process has a strong dependence on $T_{e}$. An example of this is an attachment instability. Attachment on its own does not lead to instability. However, in electronegative plasmas, attachment may be balanced not only by ionization but also by detachment. As the detachment rate is in good approximation independent of $T_{e}$, a reduction in $T_{e}$ leads to a decrease in the ionization and attachment rates but not the detachment rate. Therefore, if the attachment is mainly balanced by detachment and not ionization, the unchanged detachment rate can lead to an uncontrolled increase in the electron density and an instability.

The above description of instabilities suggests that instabilities occur in the bulk plasma and indeed contractions of the positive column of high-pressure glow discharge have been observed. Nonetheless, studies of diffuse glow discharges in the context of laser at tens to hundreds of Torr have also shown that the anode layer has a voltage current characteristic with a negative slope [44]. Such an anode layer is unstable and similarly to a cathode layer of an abnormal glow discharge susceptible of radial constriction (see e.g. [4]). The shrinkage of the anode layer finally results in the formation of anode spot(s) with high current density that can trigger an instability. Similarly, cathode spot formation has also been observed to trigger instabilities [45]. The instability criterion in these cases has been empirically related to the dissipation of a critical surface energy density.

Streamer discharges can undergo a transition to more intense spark discharges. A streamer propagates in the discharge gap, but does not necessarily connects the two opposite electrodes. However, when this happens, a conductive plasma channel is formed. The entire gap voltage is across this conductive channel which leads to an increased ionization and subsequent instability. Typical times for excessive gas heating occurs on $\sim 100 \mathrm{~ns}$ at atmospheric pressure and a streamer-to-spark transition typically 
happens on such time scales. However, instabilities in nanosecond spark discharges have also been reported. In spite the potential involvement of fast gas heating, gas heating is not a pre-requisite for the formation of a spark discharge as this could also be due to a sudden increase in the ionization rate due, for example, to stepwise ionization. An intense spark channel can transform into an even more intense arc discharge on a timescale of hundreds of microseconds if the power supply can deliver the necessary power and current.

In filamentary discharges for which the current is limited and the increase in electron density that triggers the spark formation is prevented, a streamer can develop into a transient glow discharge (see further discussion in the context of dielectric barrier discharges).

The rapid increase in electron density observed in some of these instabilities also leads to an increased importance of electron-electron Coulomb collisions. In low electron density plasmas $\left(n_{e}<<n\right)$, the EEDF is mainly determined by electron-neutral collisions and high-energy tail of the distribution is typically depleted due to the fast electron energy loss in inelastic processes. In high-density plasmas, electron-electron Coulomb collisions become important and cause a Maxwellization of the EEDF, which in turn increases the ionization rate.

\section{Discharge generation and stabilization}

\subsection{Current limitation (ballast)}
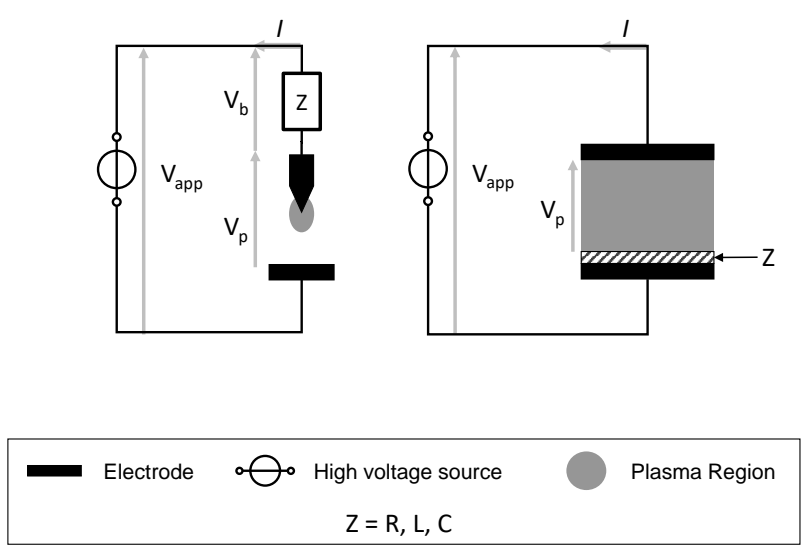

Figure 8. Schematic of lumped and distributed ballast for stabilizing discharges.

The most straightforward approach to avoid spark or arc formation is to limit the discharge current. However, as transitions and instabilities typically occur on submicrosecond up to nanosecond time scales, active feedback control approaches cannot respond quickly enough. Instead, passive electrical components in series with the 
discharge are typically used to limit the discharge current (Figure 8). The passive component in the circuit is often referred to as a ballast. For example, a resistor with a well chosen resistance value $(R)$ in series with a discharge will limit the current of the discharge because for a fixed applied voltage $\left(V_{a p p}\right)$, any increase due an incrase in plasma conductivity will lead to a drop of the voltage across the plasma $\left(V_{p}\right)$ in favor of an increasing voltage across the resistor $\left(V_{b}=I R\right)$ :

$$
V_{p}=V_{a p p}-I R
$$

Interestingly, similar effect can be achieved with capacitors and inductors. In these cases, the voltage across the plasma is:

$$
V_{p}=V_{a p p}-L \frac{d I}{d t} \quad V_{p}=V_{a p p}-\frac{1}{C} \int_{0}^{t} I_{p} d t
$$

While in the case of a resistor the voltage drop across the plasma reduces proportionally to the plasma current, in the case of the inductor the reduction is more pronounced for fast current changes and in the case of a capacitor the reduction in discharge voltage has a memory effect. Intrinsically the capacitor and inductor can have a stronger impact on transient phenomena than the resistor. The capacitor is typically used for sinusoidal excited plasmas (as it blocks any DC current) while a resistive ballast is preferred to stabilize DC operated plasmas.

The above stabilization approach consists of a lumped passive circuit component that is able to successfully stabilize DC corona discharge, dielectric barrier discharges and (low-pressure) glow discharges. However, this approach is in many cases not able to prevent plasma instabilities such as a contraction of a diffuse plasma into a filament in atmospheric pressure discharges. A more stable and effective approach in this case is the use of distributed ballasts. A distributed resistor is equivalent to the use of a resistive electrode and indeed high-pressure glow discharges have been stabilized by the use of semiconductor and liquid electrodes with finite conductivity [46, 47]. Particularly in the case of a liquid electrode, it has been shown that spark formation due to instabilities in the gas phase can still occur but the contraction of the discharge at the liquid electrode is significantly delayed due to the inability of the electrode to transport the injected charge of a high intensity spark channel because of its finite conductivity [48].

Dielectric barrier discharges (DBDs) use a similar principle. In this case the distributed capacitor is a dielectric barrier placed between the metal electrode and the discharge gap. This approach only works for AC driven or pulsed discharges as a displacement current through the non-conductive dielectric barrier is required to allow for current continuity between the conduction current in the discharge and the metal electrode connected to the electrical circuit. In this case, the voltage limitation across the discharge gap is due to charge deposition on the dielectric that reduces the local electric field in the discharge gap (see also further).

An interesting example of an inductively stabilized DBD which combines the distributed dielectric barrier with a lumped inductance in the circuit has been developed by the group of van de Sanden [49]. This approach led to a diffuse discharge which was 
not possible with the dielectric barrier only. This is a very nice example of the potential huge benefit of a detailed design of the electrical circuit on the discharge operation.

The electrical configuration of a DBD is actually very similar to an RF driven discharge between two parallel metal electrodes. In this case there is no dielectric insulating barrier but instead the plasma sheath acts as a distributed capacitor between the bulk plasma and the metal electrode. A key difference however is the physical interface of the dielectric barrier that allows for charge accumulation. This capacitive nature of the sheath is a typical feature of an RF discharge, as electrons are depleted and the inertia of the ions is too large to respond to the RF field. In this case, current continuity is achieved through displacement current caused by the movement of the sheath in the RF field. This is very different for a DC sheath were secondary emission current through the sheath enables the sustainment of the discharge [4]. However, as the 'barrier' in this case is a gas which can be ionized, this configuration is much more prone to instabilities. A breakdown of this sheath can easily occur when for example a thermal instability occurs. This enables the development of an intense filament that connects both metal electrodes. To this end researcher have investigated RF discharges with dielectric barriers [50]. Nonetheless this approach is often unpractical as the additional barrier requires higher voltages which are easy to achieve for power supplies operating in the $\mathrm{kHz}$ frequency range but needs special amplifiers or circuits for power supplies in the $\mathrm{MHz}$ frequency range $(\mathrm{RF})$.

\subsection{Transient discharges}

Another straightforward approach to prevent thermalization is to limit the discharge duration. A transient discharge can be realized by using short voltage pulses or by exploiting the self-limiting behavior of discharges such as in the DBD. Although time scales are discharge specific, the transient behavior at atmospheric pressure is typically in a time scale of $<100 \mathrm{~ns}$, in line with typical time scales for gas heating and glow-tospark transitions.

\subsubsection{Dielectric Barrier Discharge (DBD) One of the most used type of a charge-} limited, transient atmospheric-pressure plasma is the DBD. As mentioned above, DBD is characterized by an insulating material in the discharge path [9]. Dielectric materials include glass, quartz, ceramics, enamel, mica, plastics, silicon rubber or teflon and in atmospheric pressure plasmas the discharge gap is typically in the range of 0.1 to $10 \mathrm{~mm}$. The amplitude of the alternating or pulsed high voltage required to sustain a discharge is in the range $1-100 \mathrm{kV}$ and it depends on the gas and the electrode gap dimensions.

A large number of electrode arrangements exist. A so-called volume DBD is shown in Figure 9a. In this arrangement at least one of the electrodes is covered by a dielectric barrier. Volume DBDs can also be created by placing the dielectric layer in the discharge gap between the two metal electrodes, dividing the gap in two sections [51]. Packed bed DBDs have the space between the electrodes filled with pellets or spheres made of 
(a) DBD



(e) ns-pulsed discharge

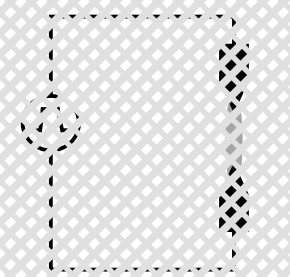

(i) Microwave microstrip resonator

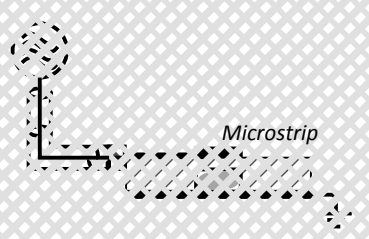

(b) Plasma jet

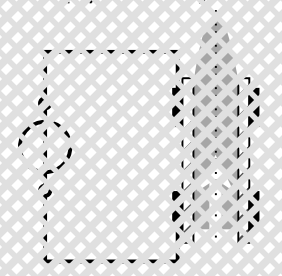

(f) CCP

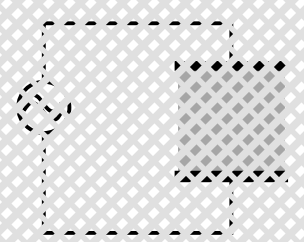

(c) DC corona



(g) $\mathrm{MHCD}$

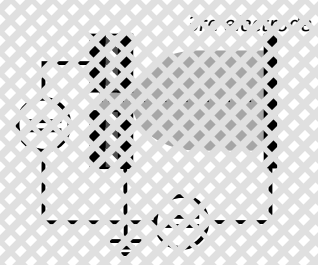

(d) Transient streamer

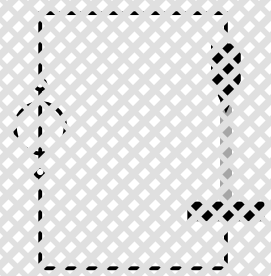

(h) Microplasma array

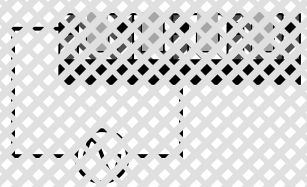

(k) Gliding arc
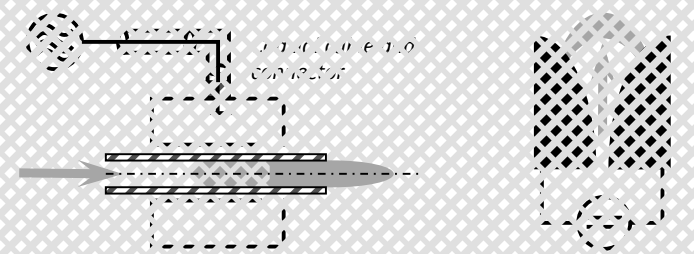

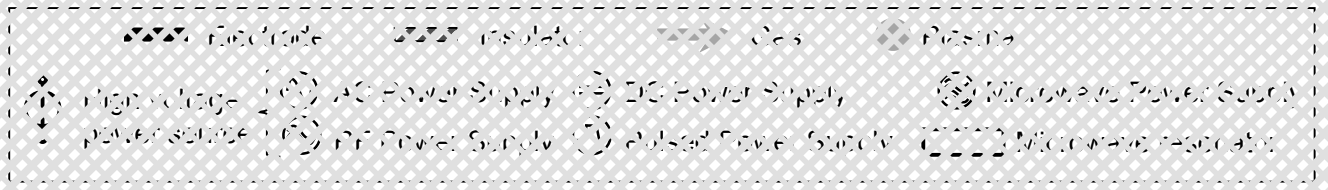

Figure 9. Schematic of different non-equilibrium atmospheric-pressure plasma sources. Examples of actual devices in operation corresponding to the electrode configurations shown can be seen in Figure 10.

dielectric or ferroelectric material. The polarization of the pellet material generates plasma in the void spaces between the pellets and on their surface. Another DBD electrode arrangment is that used in surface DBDs. Here both electrodes are in direct contact with the barrier and the plasma forms at the exposed surface electrode (see Figure 4a) [52]. Alternatively, both electrodes can be embedded inside the insulator to create a coplanar DBD in which the discharge appears in the gas above the dielectric surface. A popular atmospheric-pressure DBD structure is that of an atmosphericpressure plasma jet (Figure 9b) [53]. The gas flowing through the arrangement extends the plasma as an effluent into the surrounding gas. Whether a plasma jet can be considered as a DBD or not is mainly determined by the frequency of the applied voltage as the majority of jets consist of a dielectric tube in between the plasma and at least one of the electrodes. An overview of the large majority of different DBD arrangements and reactors including some aditional special configurations can be found in [54].

In most molecular gases but also in argon or mixtures of noble gases with molecular 
gases the streamer mechanism lead to so-called microdischarges that visually appear as filaments (see example in Figure 10a). The microdischarge development starts with a (Townsend) pre-phase (a weak, localized light spot at the anode) which can last for several 100 ns [55]. When the local electric field strength of the accumulated positive space charge in front of the anode reaches a critical value the propagation of a cathodedirected ionization wave (positive streamer mechanism) starts. Behind the streamer a transient glow-discharge like plasma channel develops [52]. The plasma decays due to the accumulation of charge carriers on the barrier surfaces and the reduction of the axial electric field strength. The microdischarge channels are spreading on the barrier surface covering a region much larger than the original channel diameter. An increase of the voltage amplitude in an AC operated, plane parallel discharge gap DBD leads to a higher number of microdischarge per active phase, but will not necessary change the amount of charge transferred to a single microdischarge.

The duration of microdischarges is determined by the gas as well as the discharge arrangement (gas gap, type, and thickness of barriers). In air at 1 atmosphere and $1 \mathrm{~mm}$ discharge gap the microdischarges have a duration between 10 to $100 \mathrm{~ns}$ with a total transferred charge of 0.1 to $10 \mathrm{nC}$. Microdischarges in argon can have a duration of a few $\mu \mathrm{s}$. The maximum current density can reach up to $1,000 \mathrm{~A} / \mathrm{cm}^{2}$. While many discharges are filamentary volume space charge and surface charges distribute plasma filaments across the entire electrode area. In many case also self-organizing patterns have been observed [51]

At frequencies in the $\mathrm{MHz}$-range the current limitation by the dielectric becomes less effective, the breakdown voltage decreases, and the discharge operation changes significantly [56]. The discharge does not behave anymore as a DBD as discussed above. The role of the barrier is no longer to induce the self-pulsing character in this case and the discharge operates in steady state. Its properties are comparable with RF discharges or capacitively coupled plasmas in the $\alpha$-mode, i.e. the electron production that sustains the discharges is in the gas phase rather than by secondary electron acceleration across the sheath ( $\gamma$-mode). Under selected conditions DBDs can be operated in a diffuse mode with uniform plasma formation as it will be discussed in subsection 6.3.

\subsubsection{Partial and nanosecond pulsed corona discharges Partial and nanosecond pulsed} discharges are typically produced in the close vicinity of a pin or a thin wire electrode, where the electric field is locally enhanced (Figure 9b) [57]. The asymmetric electrode geometry leads to a decrease in the electric field with increasing distance from the pin or wire electrode. Typically, the applied electric field value drops below the breakdown electric field at a certain distance from the electrode and if the current or voltage is limited the breakdown development is inhibited. In this case, the discharge does not extend up to the counter electrode (see example in Figure 10c), hence the name partial discharge. Discharges generated in electrode geometries that enable such a local electric field enhancement are often referred to as corona discharges [13].

In the case of DC excited corona discharges the electrode gap can be divided in two 
zones: the high electric field region in which highly energetic electrons are produced and the ion drift region in which the electric field is low and the continuity of current towards the second electrode is guaranteed by a flux of positive ions in the case of positive corona or negative ions produced by electron attachment in electronegative gases for negative corona. The morphology of the discharge depends on polarity and the applied voltage $[57,58]$. While continuous glow corona exists, many modes of DC corona consists of current pulses and the formation of streamers. An example of a DC transient negative corona discharge mode is the Trichel pulse regime, which consists of current pulses with a nearly regular repetition rate. The pulsating nature of the discharge is due to space-charge effects [59].

It is relatively straightforward to achieve conditions satisfying the Meek criterion close to a pin or thin wire electrode leading to the formation of streamers. In addition, steep voltage rise times combined with high overvoltages allows one to strongly impact the plasma parameters. In particular higher number densities of reactive species than in classical DC or AC operated discharges can be generated as the input power can be increased. At sufficient high field strengths even runaway electrons can be generated which can lead to the generation of x-rays [60, 61].

For many applications, it is crucial to increase the power deposition in the discharge while maintaining the non-equilibrium character. While overvoltages can be used, they need to be applied for a short time to prevent the streamer-to-spark transition. The short duration of the voltage pulse inhibits streamer growth before it reaches the ground electrode and a spark can be formed. An example of a nanosecond pulsed streamer discharge consisting of branching streamers with a length of several $\mathrm{cm}$ is shown in Figure 9d and 10d. Typical streamer velocities in air are in the range $10^{5}-10^{6} \mathrm{~ms}^{-1}$, depending on applied voltage and polarity. The highest velocity requires $100 \mathrm{~ns}$ to bridge a gap of $10 \mathrm{~cm}$ hence a well chosen geometry and pulse duration of tens to hundreds of nanoseconds is often able to prevent spark formation. Another approach is to cover the ground electrode with a dielectric barrier.

In recent years, there has been a large interest in the production of nanosecond pulsed glow and spark discharges in pin-pin geometries in the context of plasma assisted combustion. These discharges are typically generated with a high repetition rate in the $\mathrm{kHz}$ range (see Figure 9e and 10e). At moderate voltages these discharges, even in air are able to operate in a glow mode. However, even for voltage pulses with a duration of $10 \mathrm{ns,}$ spark discharges have been obtained, as demonstrated in Figure 10e [62]. The transition from a streamer or glow to spark in this case is shown to be enhanced by accumulated effects over multiple discharge pulses. However, fast gas heating on nanosecond time scales in air and stepwise ionization in noble gases can also cause the transition on a nanosecond timescale [63]. The exact discharge geometry, electrode properties, gas composition and potential presence of gas flow have a strong influence on the discharge mode and its instabilities. The transient spark is a non-equilibrium and highly reactive plasma, with an electron density that can be several orders of magnitude larger than glow and streamer discharges. While the gas temperature in a streamer discharge is 
close to room temperature, the gas temperature in a spark can be several hundreds up to several $1000 \mathrm{~K}$.

A special case of a transient discharge is the so-called self-pulsing transient spark discharge [64]. These discharge are mostly generated in pin-to-plane or pin-to-pin discharge arrangements with a DC-applied voltage and resistive ballast in series with the discharge. The discharge is initiated by a primary streamer, followed by a secondary streamer that develops into a spark discharge generating a short current pulse with a maximum amplitude in the range of a few Amperes. For an optimal choice of resistance, the ballast resistor causes the voltage across the discharge gap to reduce significantly when the spark is formed leading to the self-limitation of the spark discharge. This way, very short (about 10 to $100 \mathrm{~ns}$ in air) but self-pulsing spark channels are formed.

Nanosecond pulsed discharges are also used to generate plasmas in plate-plate geometries. In this case the goal is to achieve diffuse large area discharges. Higher overvoltages and short pulse durations can lead to a more diffuse discharge [65] although these effects also depend on the repetition rate and thus pre-ionization as discussed in the next section.

\subsection{Diffuse and pre-ionized discharges}

In the context of laser applications, intensive studies have been performed to generate diffuse high pressure glow discharges. Electron beam produced or stabilized plasma provide the opportunity to generate large volume diffuse discharges even at atmospheric pressure [10, 38]. An electron beam produced plasma is an example of a nonselfsustained discharge as the electrons are supplied by an external source. However, in general self-sustained plasmas are easier to implement. Unfortunately large volumes correspond to typically larger interelectrode gap distances, conditions for which at high pressure the Meek criterion is typically satisfied and streamers occur. When the volume contains pre-ionization, the streamer formation can be suppressed and a uniform discharge can be achieved.

As the tendency to form streamers and thus non-uniform plasmas is related to $\partial\left(\alpha_{e f f} / n\right) / \partial(E / n)$, gases that allow plasma formation at low voltages (He, Ne) enable to achieve a uniform breakdown. Indirect ionization processes (such as chemiionization) also slow down the ionization process for such regimes. Extensive studies of conditions with diffuse discharge operation have been performed in DBDs. The discharge uniformity is also found to relate to the ratio of $\gamma / \alpha$, with $\gamma$ the secondary electron emission coefficient. The higher the value of this ratio the higher the $p d$ value is for which a Townsend breakdown can be achieved [66]. The uniformity of the inception is also influenced by the properties of the power supply and the electrical circuit/electrode configuration.

The importance of the gas composition can be illustrated by an example: a DBD in Ar at atmospheric pressure is typically filamentary although when adding a small amount of $\mathrm{NH}_{3}$ the discharge can become diffuse. This is due to the Penning ionization 
of $\mathrm{NH}_{3}$ through Ar metastables. The breakdown electric field reduces in this case as the energy threshold for Ar metastable production is about $5 \mathrm{eV}$ lower than for ionization of Ar. In general, metastable states induce a variety of multistage or indirect ionization processes like stepwise ionization and Penning ionization which can affect the discharge formation mechanism and a Townsend discharge can be obtained at elevated pressures [66].

Typically two discharge types are achieved for diffuse discharges: very low density Townsend discharges (with negligible space charge and a homogeneous electric field) and glow discharges that develop from a Townsend breakdown after the formation of a cathode fall [66]. Glow discharges have a higher electron density and tend to be unstable often leading to a narrow stable operation window. The applied voltage waveform is thus extremely important for the stability of the diffuse operation of the glow discharge as well.

While in many cases diffuse large scale glow discharges are produced in DBD geometries, there are cases of uniform discharges generated between two metal electrodes. An example is a RF driven diffuse glow discharges in He [67], which can also be characterized as a capacitively coupled plasma (CCP, see Figure $9 \mathrm{f}$ and 10f). It is difficult to have a pure helium discharge and in many cases the dominant ion is from the impurity making Penning ionization a dominant ionization mechanism. Similarly a diffuse discharge can also be generated for nanosecond pulsed excitation in the same reactor geometry in helium. The pulse width in this case is smaller than the time scale to develop an instability ( a few $100 \mathrm{~ns}$ ). It has also been observed that high overvoltages at which the applied electric field is much higher than the breakdown voltage can lead to more diffuse discharge generation in air [65]. The detailed mechanism is in this case still under investigation.

\subsection{Flow stabilized discharges and micro-plasmas}

Besides limiting the discharge duration and/or current, additional approaches are commonly used to maintain stable non-equilibrium plasmas at atmospheric pressure. For example, many non-equilibrium atmospheric-pressure plasmas, specially those in direct contact with temperature sensitive substrates, operate in noble gases. This is because when molecular gases are used, significant amount of energy is lost via vibrational excitation of the background gas. While this may be a desirable process for some applications (e.g. in $\mathrm{CO}_{2}$ dissociation via vibrational excitation), vibrational states eventually transfer their energy into rotational and translational states ( $\mathrm{V}-\mathrm{V}$ and $\mathrm{V}-\mathrm{T}$ relaxation), thereby heating the background gas. The use of noble gases as background gas eliminates this heating mechanism. In particular, helium is widely used as a carrier gas because not only it is an atomic gas but it also has a high thermal conductivity ( 6 times that of air and $\sim 8$ times that of argon).

Other approaches aimed at preventing excessive gas heating in atmospheric-pressure plasmas include active cooling of the electrodes, cooling of the inlet gas and modulation 
of the input power. For example, modulation of the input power with a duty cycle of $20 \%$ reduces the average power dissipated in the discharge by a factor 5 , hence lowering significantly the final gas temperature. Importantly, as many reactive species have lifetimes of several $10 \mathrm{~s}$ to $100 \mathrm{~s} \mu \mathrm{s}$, if the modulation is done at a sufficiently high frequency, it may have only minimal impact on the concentration of the relevant reactive species [68].

Gas flow is an additional parameter that can be used to manage thermal loading of the device and control discharge stability [69]. For example, in plasma jets gas is forced through the plasma, contributing to the removal of heat away from the discharge and at the same time the enhancement of the transport of plasma species away from the discharge and onto the target [53, 70]. Unfortunately, as it is often the case in plasmas, an external parameter affects multiple aspects of the discharge simultaneously, and here varying the gas flow rate does not only control heat removal but also affect mixing of gases and residence time of species, which will affect the overall performance of the plasma source (see Figures $4 \mathrm{~b}$ and $9 \mathrm{~b}$ ).

Despite being susceptible to chaotic operation and very sensitive to environmental conditions, plasma jets have become popular devices among scientists working in the field of material processing and medical applications as these devices are well suited for the delivery of localized treatments $[53,70]$. Different electrode configurations and operation frequencies ( $\mathrm{DC}, \mathrm{kHz}, \mathrm{MHz}$ and $\mathrm{GHz}$ ) have been reported in the literature and in nearly all cases a noble gas is used as a carrier gas to generate a channel in which ionization takes place preferentially. Although the visual appearance of most jets is quite similar, the underpinning physics can be quite different. Long plasma plumes observed in $\mathrm{kHz}$ and nanosecond pulsed jets are often guided streamers confined in the noble gas channel created by the gas flow $[82,83]$. This confinement is due to the spatial gradient of the $\alpha$ coefficient that drastically reduces with increasing air concentration at the edge of the noble gas channel and the electrostatic focusing caused by negative ions (e.g. $\mathrm{O}_{2}^{-}$) in the mixing region. As shown in Figure 10b, the composition of the ambient in which the jet operates has a strong influence on the appearance and properties of these jets.

Thermal dissipation can also be enhanced by having a large surface to volume ratio and as a result many non-equilibrium atmospheric-pressure plasma sources rely on creating small discharges in parallel (arrays). The small size not only helps in maintaining a large surface to volume ratio but also favors operation closer to the minimum of the Paschen curve. Advances and popularization of microfabrication techniques in the 1990s triggered the development of the so-called microplasmas and microplasma arrays, reviving the interest in high-pressure discharges. Many different electrode configurations exists and several review papers have been written on this topic $[19,71,72,73]$.

Figures $9 \mathrm{~g}$ and $10 \mathrm{~g}$ depict a 3 -electrode DC plasma in which a microhollow cathode discharge is used as a virtual "plasma cathode" to maintain a larger volume stable plasma between the microhollow cathode and a third positively biased electrode. In 
this device, the plasma cathode reduces the cathode fall and allows the generation of a larger and more stable plasma [74, 80]. DC microdischarges have been widely studied and are appealing for their relative simplicity. Operation in DC fields, however, requires large breakdown voltages and the constant bombardment of the cathode required for DC operation limits the lifetime of these devices even when low-sputtering yield materials are used. Nonetheless, this limitation of DC microplasmas can be turned into a strength in applications that use liquids as an electrode because eroded material is easily replenished. DC microplasmas have therefore received renewed attention in applications involving plasma-liquid interactions with applications for both analytical and electrochemical purposes.

Arrays of DC microdischarges have been operated without the need of external ballast resistors when plasmas are operated in the abnormal glow mode. In this mode each discharge displays a positive differential resistance, making parallel operation possible. Uniformity of the arrays, however, is often affected by the current distribution across thin film electrodes, which ultimately limits the number of microdischarges that can be operated in parallel. AC and pulsed excitation, however, enable operation of larger arrays (see e.g. Figures 9h). An example of a microplasma array operating at $135 \mathrm{kHz}$ is shown in Figure 10h. In particular, the image depicts a $25 \mathrm{~W}$ VUV (172nm) lamp consisting of interlaced arrays of microplasmas operating in $70 \% \mathrm{Xe} / \mathrm{Ne}$ mixture at 550 Torr (at $300 \mathrm{~K})$.

Microwave operated plasmas are well-known in the field of low pressure plasmas as they enable high power absorption resulting in high electron densities. In case of higher pressures the power deposition criterion is even better matched and an efficient plasma generation is possible [8]. Also non-equilibrium and small-scale microwave driven plasmas can be generated at atmospheric pressure. Figure 10i shows an example of an array of microwave microplasmas operating in argon at 100 Torr. These MW microplasmas typically use guides such as microstrip lines, coaxial structures or strip lines instead of lumped circuit elements, resulting in compact and robust designs that minimize radiation losses and interference. Interestingly, MW microplasmas selfstabilize, preventing the transition into an arc. The lower sheath voltages encountered in these high frequency plasmas result in efficient discharges and long-lived devices. Although driven at MW frequencies, these microplasmas can be viewed as high frequency CCPs, because the actual plasma dimensions are much smaller than the wavelength of the excitation frequency. Larger atmospheric pressure MW plasmas are typically sustained by surfatrons (see Figures $9 \mathrm{j}$ and $10 \mathrm{j}$ ) or slotted waveguides, devices that have also been adapted to create microplasmas [68, 88, 89, 90].

Figures $9 \mathrm{k}$ and $10 \mathrm{k}$ show the gliding arc operating in atmospheric air. Gliding arcs are another example of plasma sources that depend on gas flow to reach a nonequilibrium state. Here, the discharge is generated across two diverging electrodes. Initially it ignites at the position of the shortest distance between the electrodes and forms a quasi-thermal plasma. This discharge then moves upwards (glides) between the electrodes due to the applied gas flow and/or the buoyancy force. The diverging 
geometry of the electrodes causes the length of the plasma to increase and the power supply eventually is not able to deliver enough input energy to maintained the increasingly longer discharge. As a result, the discharge intensity decreases and the original quasi-thermal plasma is converted into a non-equilibrium plasma before the plasma extinguishes and a new arc is formed [86, 87].

While heat losses are important to prevent an excessive rise of the gas temperature and maintain the non-equilibrium character in atmospheric-pressure plasmas, losses of charged and metastable species also play an important role in maintaining discharge stability, for example, by reducing the effective ionization rate. Many of the techniques described above (e.g. use of helium, forced flows and large surface to volume ratios) simultaneously affect both heat and particle losses, thereby contributing in more than one way to the non-equilibrium nature of the discharge.

\section{Conclusion and Outlook}

An extremely rich variety of non-equilibrium atmospheric-pressure plasmas exists ranging from Townsend discharges to spark discharges that span a range of ionization degrees of 10 orders of magnitude. This paper attempts to provide an introductive overview of the main aspects of these plasmas but is far from being complete. We did for example not deal with thermal high-pressure plasmas that in spite their name have often significant non-equilibrium effects as has been discussed in the related foundations article on this topic.

While the ability to generate stable low temperature, high-pressure plasmas is well established, new operation approaches and plasma regimes are continuously being explored. The physics and chemistry of these complex discharges is often much more unexplored compared to their low pressure counterparts. A strong coupling between plasma kinetics, heat transfer, chemistry and fluid dynamics makes these plasmas an intrinsic multidisciplinary problem. However, these particular properties and unique conditions underpin many applications that would not be possible by other technologies. In addition, the self-organizing nature and filamentation of high-pressure plasmas leading to significant spatial and temporal gradients in plasma properties provides many additional challenges and interesting scientific questions. The high collisionality and non-equilibrium nature of the plasma are also strongly complicating factors for the interpretation of diagnostics.

New applications continuously drive to explore the interaction of plasma with previously unexplored substrates including living tissue and complex solutions, which only increases this challenge. While detailed characterization starts to emerge for a few atmospheric-pressure plasma sources, the plasma properties and kinetics for higher gas densities, including liquids and supercritical fluids remain relative unexplored.

Despite their large history, recent developments in state-of-the-art diagnostics and

modeling capabilities will provide the unique opportunity to tackle these challenges and move the important field of non-equilibrium high-pressure plasmas towards new 
(a) DBD

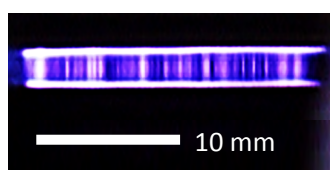

(e) ns-pulsed discharge

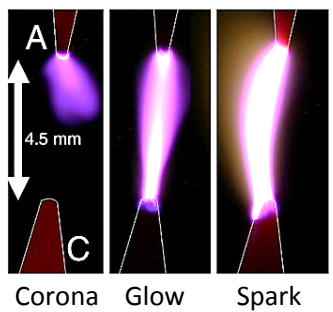

(i) Microwave microstrip resonator

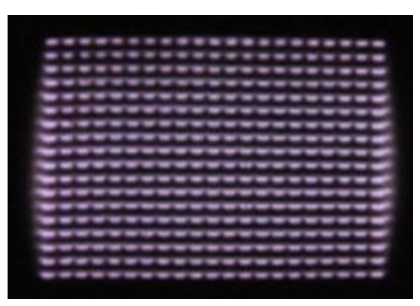

(b) Plasma jet

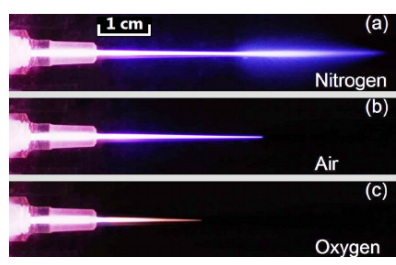

(f) CCP

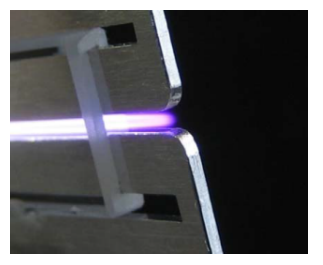

(j) Surfatron plasma

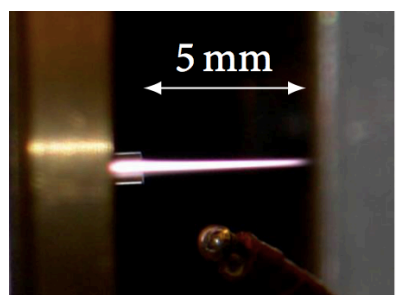

(c) DC corona

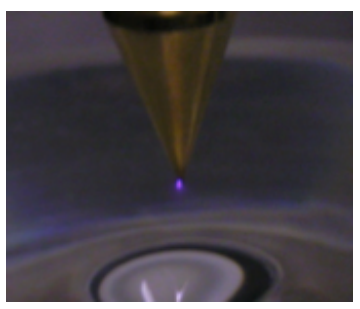

(g) MHCD

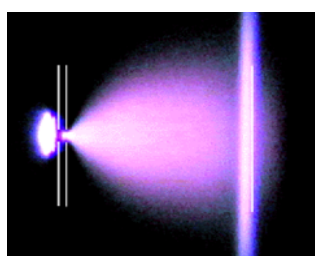

(k) Gliding arc

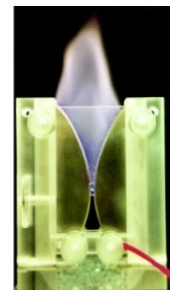

(d) Transient streamer

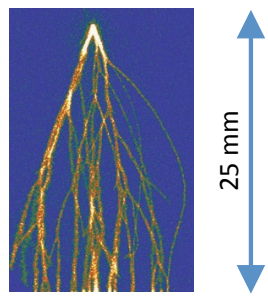

(h) Microplasma array



Figure 10. Examples of high-pressure non-equilibrium plasmas. a) Parallel-plate filamentary dielectric barrier discharge in atmospheric pressure air. b) Atmospheric pressure He plasma jet propagating in three different ambient gases: nitrogen, air, and $\mathrm{O}_{2}$. Reprinted from [76], with the permission of AIP Publishing. c) DC corona in atmospheric air. d) $50 \mu s$ exposure time image of a transient streamer in air. Reprinted from [77], with permission of IOP Publishing. e) 10-nanosecond pulsed discharge in air operating in corona, glow and spark regimes at $5 \mathrm{kV}, 5.5 \mathrm{kV}$ and $6 \mathrm{kV}$, respectively. Reprinted from [78], with permission of IOP Publishing. f) Capacitively coupled RF $(13.56 \mathrm{MHz})$ plasma sustained in helium at atmospheric pressure. Reprinted from [79], with permission of IOP Publishing. g) 3-electrode DC plasma in argon at 160 Torr in which a microhollow cathode (left electrodes) serves as a virtual cathode for a DC discharge sustained between the virtual cathode and an anode (right electrode). Reprinted from [80], with the permission of AIP Publishing. h) Front view of a $10 \mathrm{~cm} \mathrm{x}$ $10 \mathrm{~cm} \mathrm{Xe} \mathrm{Xe}_{2}$ VUV (172nm) lamp consisting of an array of encapsulated microplasmas operating in $70 \% \mathrm{Xe} / \mathrm{Ne}$ admixture at 550 Torr. Reprinted from [81], with the permission of AIP Publishing. i) MW microplasma array operating in 100 Torr argon. Reprinted from [84], with the permission of IOP Publishing. j) $2.45 \mathrm{GHz}$ surfatron operated in argon at atmospheric pressure. Reprinted with permission from [85]. k) Gliding arc operating in ambient air. Reprinted from [86], with the permission of AIP Publishing.

exciting science and unique applications that will without doubt be of great benefit for 
our society.

\section{Acknowledgments}

PJB acknowledges the support of the Center on Control of Plasma Kinetics of the United States Department of Energy Office of Fusion Energy Science (DE-SC0001319), the National Science Foundation (PHY 1500135) and a Department of Energy Early Career Research Award (DE-SC0016053). FI acknowledges the support of the Loughborough University Center for doctoral training on Gas Plasma Interactions with Organic Liquids and the UK Engineering and Physical Sciences Research Council (EP/I032193/1). RBr acknowledges the support of the German Research Foundation (DFG, Grant BR 4133/4$1)$.

\section{References}

[1] Samukawa S, Hori M, Rauf S, Tachibana K, Bruggeman P, Kroesen G, Whitehead JC, Murphy AB, Gutsol AF, Starikovskaia S, Kortshagen U, Boeuf JP, Sommerer TJ, Kushner MJ, Czarnetzki U and Mason N 2012 J. Phys. D: Appl. Phys. 45253001

[2] Kogelschatz U 2003 Plasma Chem. Plasma Proc. 231 1-46

[3] Adamovich I, Baalrud S D, Bogaerts A, Bruggeman P J, Cappelli M, Colombo V, Czarnetzki U, Ebert U, Eden J G, Favia P, Graves D B, Hamaguchi S, Hieftje G, Hori M, Kaganovich I D, Kortshagen U, Kushner M J, Mason N J, Mazouffre S, Thagard S M, Metelmann H-R, Mizuno A, Moreau E, Murphy A B, Niemira B A, Oehrlein G S, Petrovic Z L, Pitchford L C, Pu Y-K, Rauf S, Sakai O, Samukawa S, Starikovskaia S, Tennyson J, K Terashima, Turner M M, Sanden M C M van de and Vardelle A 2017 J. Phys. D: Appl. Phys. 50323001

[4] Lieberman MA and Lichtenberg AJ 2005 Principles of Plasma Discharges vol 2nd (New Jersey: John Wiley and Sons Inc.)

[5] Finkelnburg W and Maecker H 1956 Electric Arcs and Thermal Plasmas in Encyclopedia of Physics XXII, ed. S. Flügge, 307 (Berlin: Springer).

[6] Boulos M I, Fauchais P and Pfender E Thermal Plasmas - Fundamentals and Applications Vol. 1 1994 /New York: Plenum Press)

[7] Raizer YP 1991 Gas discharge physics, 2nd edition (Berlin: Springer)

[8] Meichsner J, Schmidt M, Schneider R and Wagner HE (Editors) 2012 Nonthermal Plasma Chemistry and Physics (Boca Raton: CRC Press Taylor \& Francis Group)

[9] Becker KH, Kogelschatz U, Schoenbach KH and Barker RJ (editors) 2005 Non-equilibrium air plasmas at atmospheric pressure (Bristol: Institute of Physics Publishing)

[10] Fridman A 2008 Plasma Chemistry (Cambridge University Press)

[11] Raether H 1964 Electron avalanches and breakdown in gases (London: Butterworths)

[12] Loeb LB and Meek JM 1941 The mechanism of the electric spark (Stanford University Press; London: Humphrey Milford)

[13] Nasser E 1971 Fundamentals of gaseous ionization and plasma electronics (New York: WileyInterscience)

[14] Meek JM and Craggs JD, 1978 Electrical breakdown of gases (New York: Wiley, New York)

[15] Loeb LB and Meek JM, 1941 The Mechanism of the Electric Spark (Stanford, CA: Stanford University Press)

[16] Paschen F 1889 Ann. Phys. 273 69-96

[17] Walsh J L, Zhang Y T, Iza F and Kong M G 2008 Appl. Phys. Lett. 93221505

[18] Go DB and Venkattraman A 2014 J. Phys. D: Appl. Phys. 47503001 
[19] Iza F, Kim GJ, Lee SM, Lee JK, Walsh JL, Zhang YT and Kong MG 2008 Plasma Process. Polym. $5322-44$

[20] Popov N 2011 J. Phys. D: Appl. Phys 44285201

[21] Xu DA, Shneider MN, Lacoste DA and Laux CO 2014 J. Phys. D: Appl. Phys. 47235202

[22] Kawamura E, Vahedi V, Lieberman M A and Birdsall C K 1999 Plasma Sources Sci. Technol. 8 R45

[23] Donko Z, Schulze J, Heil B G and Czarnetzki U 2009 J. Phys. D: Appl. Phys. 42025205

[24] Moreau E 2007 J. Phys. D: Appl. Phys. 40 605-36

[25] Taglioli M, Shaw A, FitzPartick B, Neretti G, Seri P, Borghi CA and Iza F 2016 Plasma Sources Sci. Technol. 25 06LT01

[26] Segawa T, Yoshida H, Takekawa S, Jukes T and Choi K-S 2008 ASME - Fluids Engineering Division Summer Meeting 465-72

[27] Schäfer J, Bonaventura Z, Foest R et al. 2015 Europ. Phys. J. Appl. Phys. 7120804

[28] Ghasemi M, Olszewski P, Bradley J W and Walsh J L 2013 J. Phys. D: Appl. Phys. 46052001

[29] Xu D A, Lacoste D A, Rusterholtz D L, Elias P-Q, Stancu G D and Laux C O 2011 Appl. Phys. Lett. 99121502

[30] Bruggeman P, Iza F, Lauwers D, Gonzalvo YA 2010 J. Phys. D: Appl. Phys. 431012003

[31] Zhu XM and Pu YK 2009 J. Phys. D: Appl. Phys. 43015204

[32] Benedikt J, Hecimovic A, Ellerweg D and Keudell A von 2012 J. Phys. D: Appl. Phys. 45403001

[33] Bruggeman PJ and Brandenburg R 2013 J. Phys. D: Appl. Phys. 46464001

[34] Ono R 2016 J. Phys. D: Appl. Phys. 49083001

[35] Nijdam S, van Veldhuizen E, Bruggeman P and Ebert U 2012 An introduction to nonequilibrium plasmas at atmospheric pressure in Parvulescu V I, Magureanu M and Lukes P (eds.) Plasmas in Liquids and Gas-Liquid Environments (Weinheim: Wiley)

[36] Brenning N, Axnas I, Nilsson O and Eninger J E 1997 IEEE Trans. Plasma Sci. 25 83-8

[37] Pancheshnyi S 2005 Plasma Sources Sci. Technol. 14645

[38] Palmer AJ 1974 Appl. Phys. Lett. 253 138-140

[39] Bruggeman PJ and Leys C 2009 J. Phys. D: Appl. Phys. 42053001

[40] Lesaint O 2016 J. Phys. D: Appl. Phys. 49144001

[41] Bonifaci N, Denat A and Atrazhev V 1997 J. Phys. D: Appl. Phys. 302717

[42] Bruggeman P, Walsh JL, Schram DC, Leys C and Kong MG 2009 Plasma Sources Sci. Technol. 18045023

[43] Castanos-Martinez E, Moisan M, and Kabouzi Y 2009 J. Phys. D Appl. Phys. 42012003

[44] Akishev Y, Grushin M, Kochetov I, Karalnik V, Napartovich A and Trushkin N 2005 Plasma Sources Sci. Technol. 14 S18-S25

[45] Fujiwara T, Saot T, Sekikawa J and Yamada H 1994 J. Phys. D: Appl. Phys. 27 826-9

[46] Laroussi M 2002 Trans. Plasma Sci. 30158

[47] Laroussi M, Lu XP and Malott CM 2003 Plasma Sources Sci. Technol. 12 53-56

[48] Bruggeman P, Graham L, Degroote J, Vierendeels J and Leys C 2007 J. Phys. D: Appl. Phys. 40 4779-86

[49] Aldea E, Peeters P, De Vries H and Van De Sanden MCM 2005 Surface Coat. Technol. 200 1-4, $46-50$

[50] Shi JJ and Kong MG (2007) Appl. Phys. Lett. 90111502

[51] Kogelschatz U 2010 J. Phys.: Conf. Ser. 257012015

[52] Pietsch G J and Gibalov V I 2012 Plasma Sources Sci. Technol. 21024010

[53] Lu X, Laroussi M and Puech V 2012 Plasma Sources Sci. Technol. 21034005

[54] Brandenburg R 2017 Plasma Sources Sci. Technol. 26053001

[55] Kozlov K V, Wagner H-E, Brandenburg R and Michel P 2001 J. Phys. D: Appl. Phys. 343164

[56] Kunhardt E E 2000 IEEE Trans. Plasma Sci. 28 189-200

[57] Goldman M, Goldman A and Sigmond RS 1985 Pure \&J Appl. Chem. 57 9, 1353-1362

[58] Chang JS, Lawless PA, and Yamamoto T 1991 IEEE Trans. Plasma Sci. 196 1152-1166. 
[59] Loeb LB 1952 Phys. Rev. 86256

[60] Stankevich Y and Kalinin V 1967 Sov. Phys.-Dokl. 12 1042-3

[61] Noggle R C, Krider E P and Wayland J R 1968 J. Appl. Phys. 39 4746-8

[62] Pai DZ, Lacoste DA, and Laux CO 2010 Plasma Sources Sci. Technol. 19065015

[63] Aleksandrov NL, Bazelyan EM, Gorunov AYu and Kochetov IV, 1999 J. Phys. D: Appl. Phys. 32 2636

[64] Janda M, Martisovits V, and Machala Z 2011 Plasma Sources Sci. Technol. 20035015

[65] Liu C, Dobrynin D and Fridman A, 2014 J. Phys. D: Appl. Phys. 47252003

[66] Massines F, Gherardi N, Naude N and Segur P 2009 Eur. Phys. J. Appl. Phys. 4722805

[67] Shi JJ and Kong MG (2005) J. Appl. Phys. 97023306

[68] van Gessel AFH, Alards KMJ and Bruggeman PJ 2013 J. Phys. D: Appl. Phys. 46265202

[69] Goossens O, Callebaut T, Akishev Yu S, Napartovich AP, Trushkin NI and Leys C 2002 IEEE Trans. Plasma Science 30 176-177

[70] Laroussi M and Akan A 2007 Plasma Process. Polym. 4 777-88

[71] Becker KH, Schoenbach KH and Eden JG 2006 J. Phys. D: Appl. Phys. 39 R55-R70

[72] Tachibana K 2006 Trans. Electrical and Electronic Eng. 1 145-155

[73] Schoenbach K H and Becker K 2016 Eur. Phys. J. D 7029

[74] Sugawara M, Murata K, Ohshima T, Motohashi K and Kobayashi T 1981 J. Phys. D: Appl. Phys. 14 L137

[75] Antao D S, Staack D A, Fridman A and Farouk B 2009 Plasma Sources Sci. Technol. 18035016

[76] Xian Y B, Zou D D, Lu X P, Pan Y and Ostrikov K 2013 Appl. Phys. Lett. 103094103

[77] van Veldhuizen E M and Rutgers W R J. Phys. D: Appl. Phys. 352169

[78] Pai D Z, Stancu G D, Lacoste D A and Laux C O 2009 Plasma Sources Sci. Technol. 18045030

[79] Niemi K, Reuter S, Schaper L, Knake N, Gathen V S der and Gans T 2007 J. Phys.: Conf. Ser. 71012012

[80] Stark R H and Schoenbach K H 1999 J. Appl. Phys. 852075

[81] Park S-J, Herring C M, Mironov A E, Cho J H and Eden J G 2017 APL Photonics 2041302

[82] Boeuf JP,Yang LL and Pitchford LC 2012 J. Phys. D: Appl. Phys. 46015201

[83] Naidis GV 2011 J. Phys. D: Appl. Phys. 44215203

[84] Hopwood J, Hoskinson A R and Gregorio J 2014 Plasma Sources Sci. Technol. 23064002

[85] van Gessel A.F.H., 2013 Laser diagnostics on atmospheric pressure plasma jets, PhD Thesis, Eindhoven University of Technology

[86] Kalra C S, Cho Y I, Gutsol A, Fridman A and Rufael T S 2005 Review of Scientific Instruments 76025110

[87] Czernichowski A 1994 Pure and Appl. Chem. 66 1301-1310

[88] Moisan M, and Zakrzewski Z 1991 J. Phys. D Appl. Phys. 24 (7): 1025-1048

[89] Narendra J J, Grotjohn T A and Asmussen J 2008 Plasma Sources Sci. Technol. 17035027

[90] Suzuki H, Nakano S, Itoh H, Sekine M, Hori M, and Toyoda H 2015 Jpn. J. Appl. Phys. 55 01AH09 